\title{
Ionic Liquid Membranes for Carbon Dioxide Separation
}

\author{
Christina R. Myers*, Jeffery B. Ilconich**, David R. Luebke, *** and \\ Henry W. Pennline**** \\ United States Department of Energy \\ National Energy Technology Laboratory \\ P.O. Box 10940, Pittsburgh, PA 15236-0940 \\ *Phone: (412)386-5756, Email: christina.myers@netl.doe.gov \\ **Phone: (412)386-6862, Email: jeffery.ilconich@netl.doe.gov \\ ***Phone: (412)386-4118, Email: david.luebke@netl.gov \\ ****Phone: (412)386-6013, Email: henry.pennline@netl.doe.gov
}

Recent scientific studies are rapidly advancing novel technological improvements and engineering developments that demonstrate the ability to minimize, eliminate, or facilitate the removal of various contaminants and green house gas emissions in power generation. The Integrated Gasification Combined Cycle (IGCC) shows promise for carbon dioxide mitigation not only because of its higher efficiency as compared to conventional coal firing plants, but also due to a higher driving force in the form of high partial pressure. One of the novel technological concepts currently being developed and investigated is membranes for carbon dioxide $\left(\mathrm{CO}_{2}\right)$ separation, due to simplicity and ease of scaling. A challenge in using membranes for $\mathrm{CO}_{2}$ capture in IGCC is the possibility of failure at elevated temperatures or pressures. Our earlier research studies examined the use of ionic liquids on various supports for $\mathrm{CO}_{2}$ separation over the temperature range, $37^{\circ} \mathrm{C}-300^{\circ} \mathrm{C}$. The ionic liquid, 1-hexyl3methylimidazolium Bis(trifluoromethylsulfonyl)imide, ([hmim] $\left.\left[\mathrm{Tf}_{2} \mathrm{~N}\right]\right)$, was chosen for our initial studies with the following supports: polysulfone (PSF), poly(ether sulfone) (PES), and cross-linked nylon. The PSF and PES supports had similar performance at room temperature, but increasing temperature caused the supported membranes to fail. The ionic liquid with the PES support greatly affected the glass transition temperature, while with the PSF, the glass transition temperature was only slightly depressed. The cross-linked nylon support maintained performance without degradation over the temperature range $37-300^{\circ} \mathrm{C}$ with respect to its permeability and selectivity. However, while the cross-linked nylon support was able to withstand temperatures, the permeability continued to increase and the selectivity decreased with increasing temperature. Our studies indicated that further testing should examine the use of other ionic liquids, including those that form chemical complexes with $\mathrm{CO}_{2}$ based on amine interactions. The hypothesis is that the performance at the elevated temperatures could be improved by allowing a facilitated transport mechanism to become dominant. Several amine-based ionic liquids were tested on the cross-linked nylon support. It was found that using the amine-based ionic liquid did improve selectivity and permeability at higher temperature. The hypothesis was confirmed, and it was determined that the type of amine used also played a role in facilitated transport. Given the appropriate aminated ionic liquid with the cross-linked nylon support, it is possible to have a membrane capable of separating $\mathrm{CO}_{2}$ at IGCC conditions. With this being the case, the research has expanded to include separation of other constituents besides $\mathrm{CO}_{2}\left(\mathrm{CO}, \mathrm{H}_{2} \mathrm{~S}\right.$, etc.) and if they play a role in membrane poisoning or degradation. This communication will discuss the operation of the recently fabricated ionic liquid membranes and the impact of gaseous components other than $\mathrm{CO}_{2}$ on their performance and stability. 


\section{NATIONAL ENEPCY TECHNOLOCY LABOPATOPY}

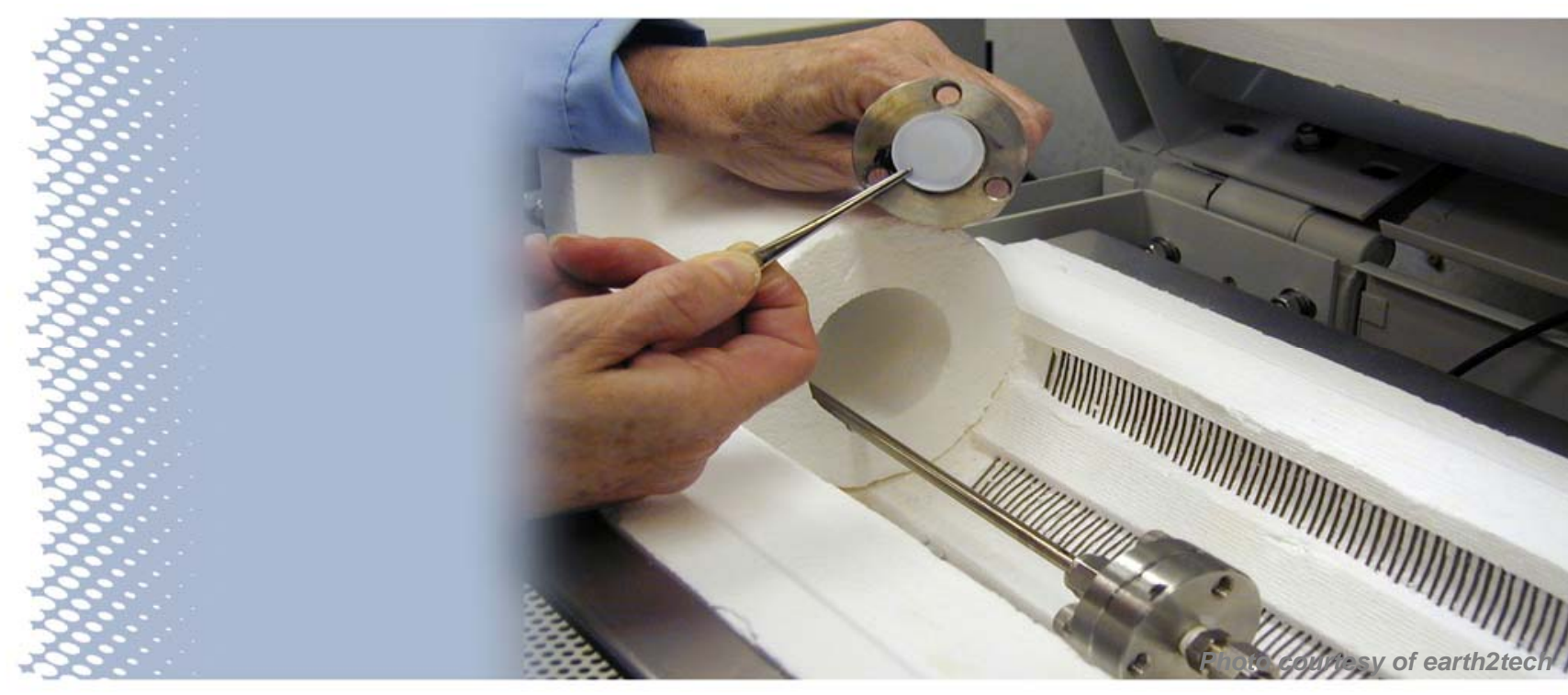

Ionic Liquid Membranes for Carbon Dioxide Separation

Christina Myers, Jeffery Ilconich, David Luebke and Henry Pennline 


\section{$R \& D$ Focus is on $\mathrm{CO}_{2}$}

\section{United States Greenhouse Gas Emissions}

(Equivalent Global Warming Basis)

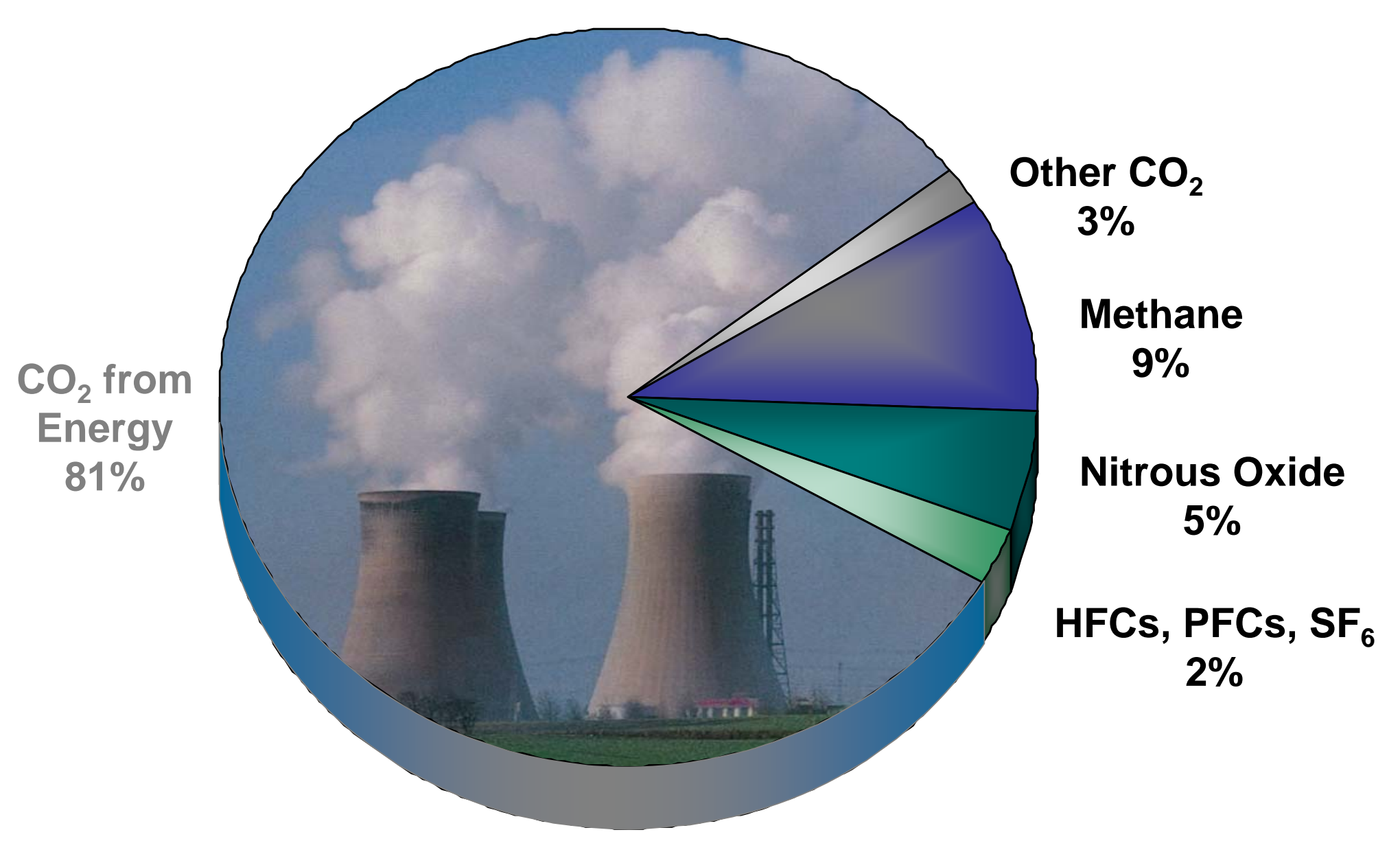




\section{Greenhouse Gas Emissions}

- Anthropogenic $\mathrm{CO}_{2}$

- One-third from power generation point sources

- Majority of point sources burn coal

- Reduction $\mathrm{CO}_{2}$ emissions

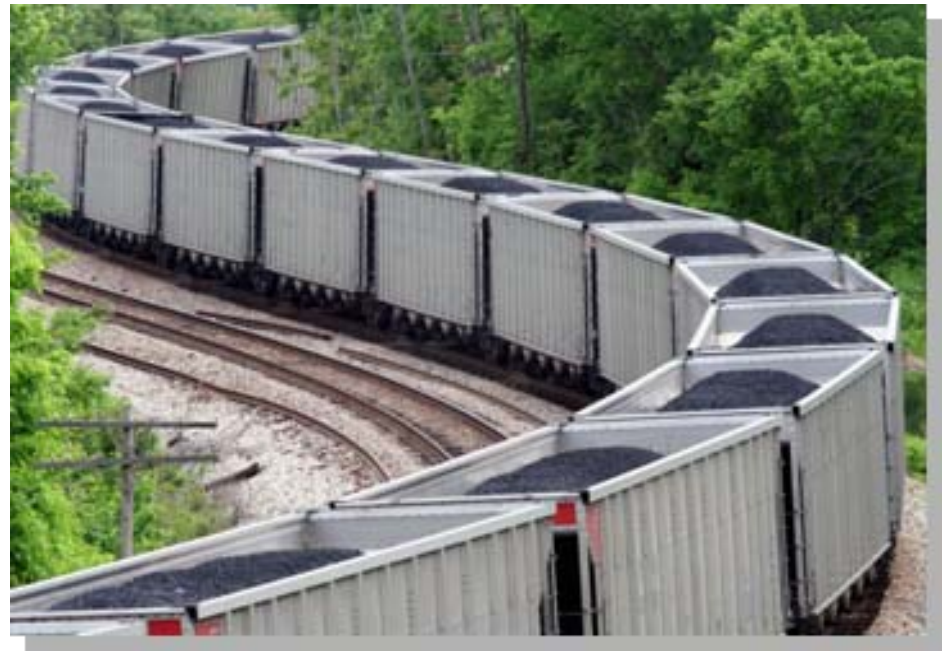

- Switch to renewable energy

- Increase process efficiency

- Use lower-carbon content fuels or sources

- Capture and sequester $\mathrm{CO}_{2}$ 


\section{Pulverized Coal Combustion (PCC-Flue Gas)}

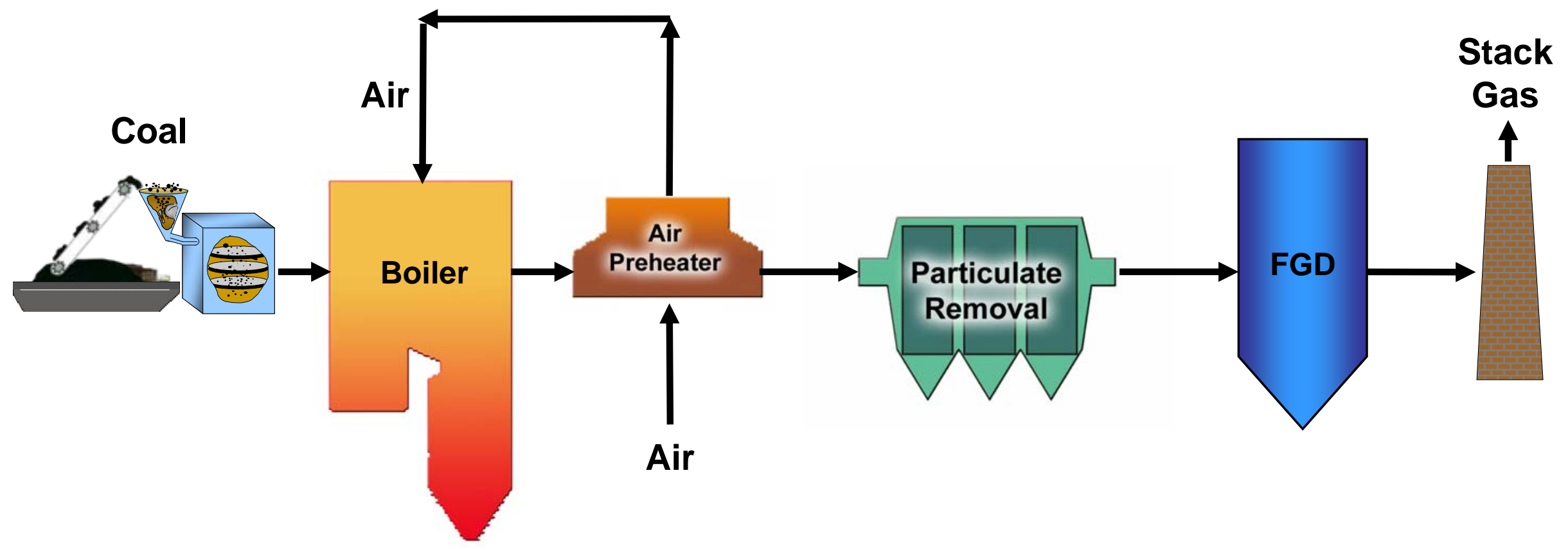




\section{Critical Separation Challenges Post- versus Pre-Combustion}

- Post-combustion (PCC)

- Low pressure + dilute volume = high volume of gas

- Trace impurities in flue gas $\rightarrow$ reduce $\mathrm{CO}_{2}$ adsorbing processes

- Compressing captured $\mathrm{CO}_{2} \rightarrow$ large parasitic load

- Pre-combustion (IGCC)

- $\mathrm{CO}_{2}$ concentration (40 volume \%)

- High pressure and temperature

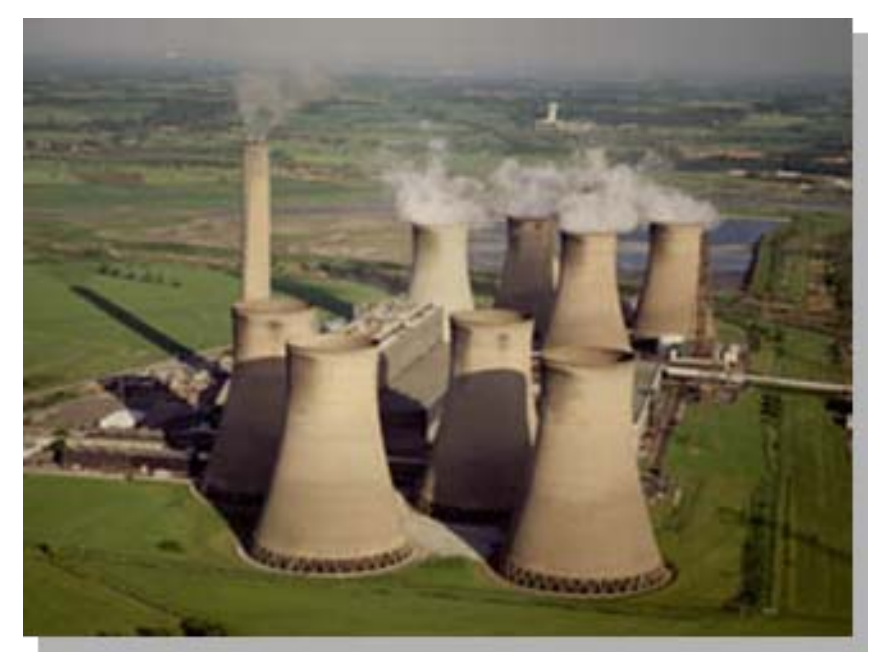




\section{IGCC with $\mathrm{CO}_{2}$ Capture}

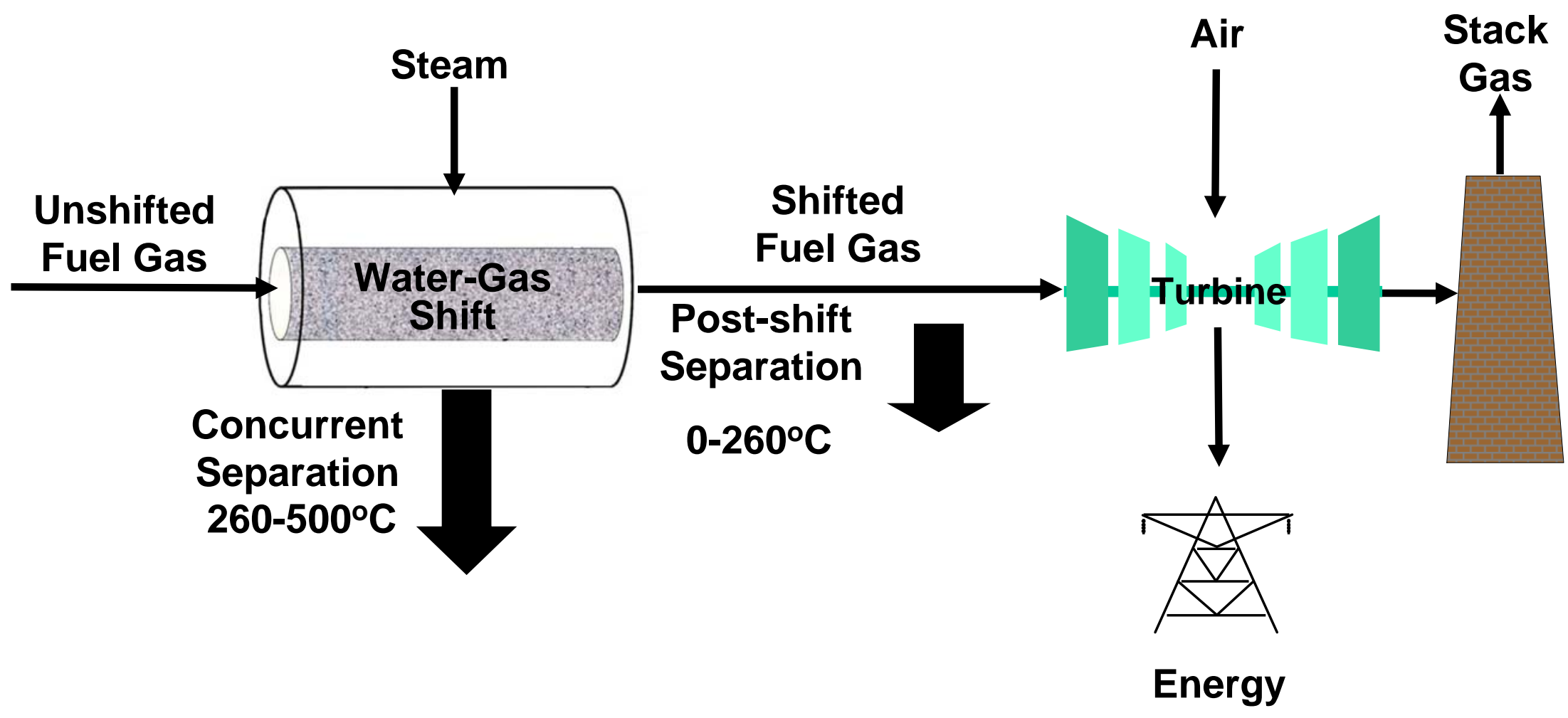




\section{Water-Gas Shift}

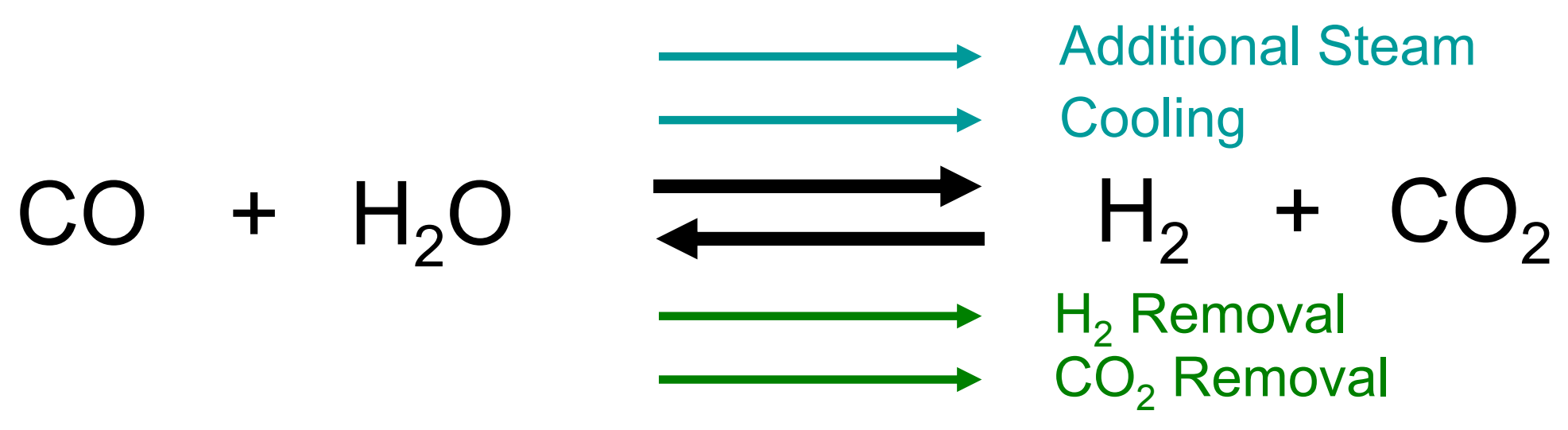

- Conventional

- Cooling to $260^{\circ} \mathrm{C}$

- Additional Steam

- Improvement through Separation

- Cooling Only to Separation Temperature

- Only Stoichiometric Steam Required 


\section{Supported Liquid Membranes}

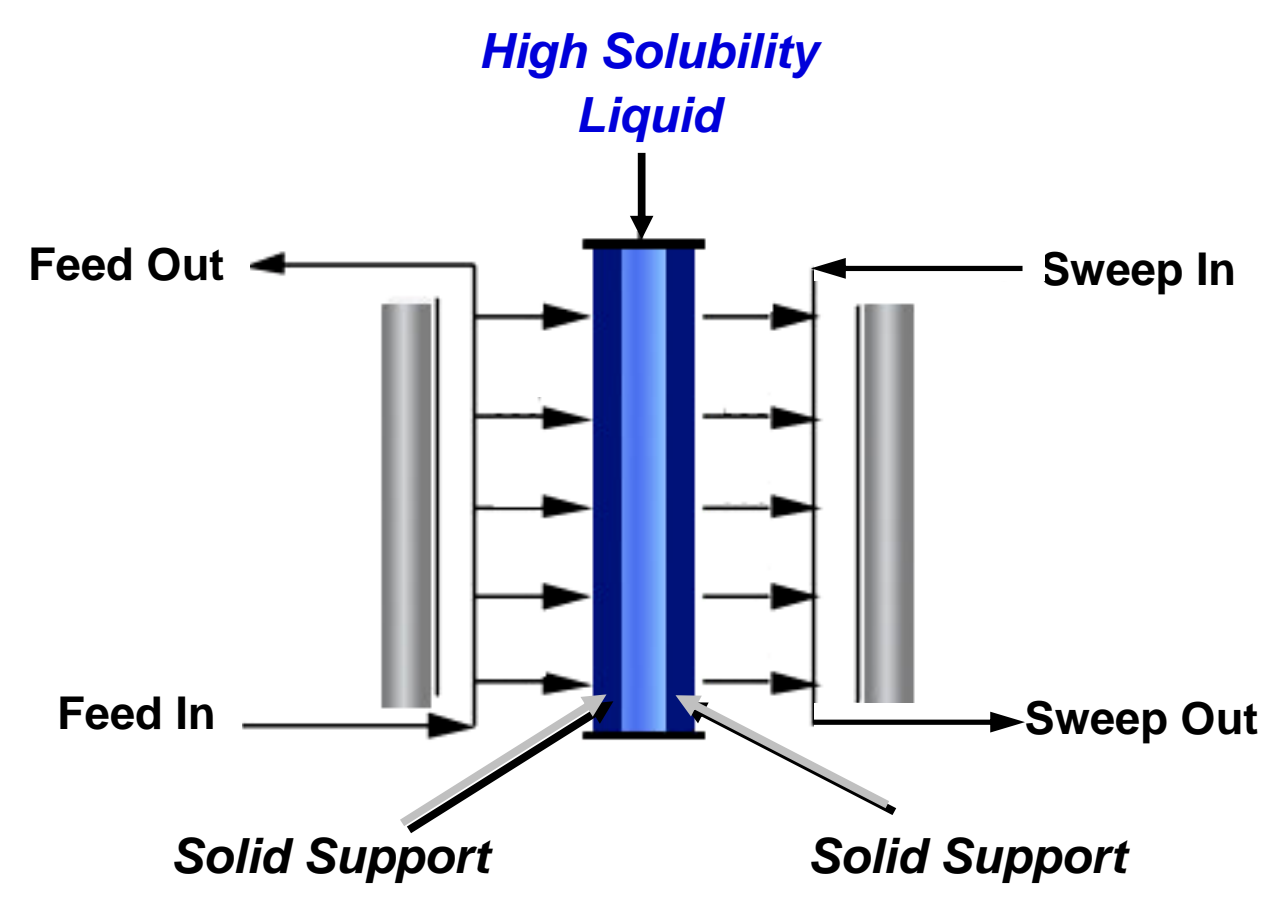

- High liquid phase diffusivity increases permeability

- Potential to add complexes increasing $\mathrm{CO}_{2}$ solubility

- Problems

- Evaporation of liquid

- Blowout 


\section{Ionic Liquids: A Solution to Evaporative Failure}

Cations
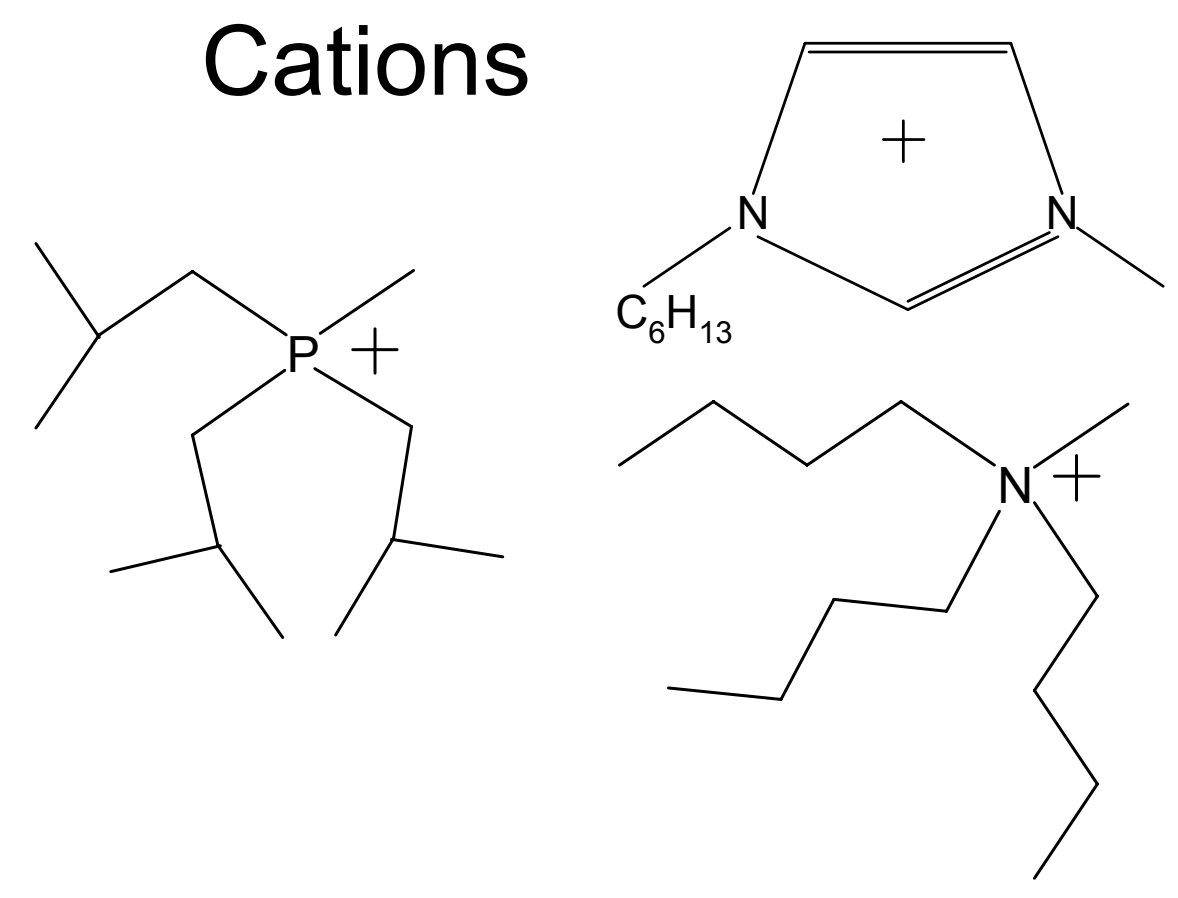

- Negligible Vapor Pressure

- Thermally Stable above $200^{\circ} \mathrm{C}$

- High $\mathrm{CO}_{2}$ Solubility Relative to $\mathrm{H}_{2}, \mathrm{~N}_{2}$, and $\mathrm{CH}_{4}$ 


\title{
Several Fabrication Options
}

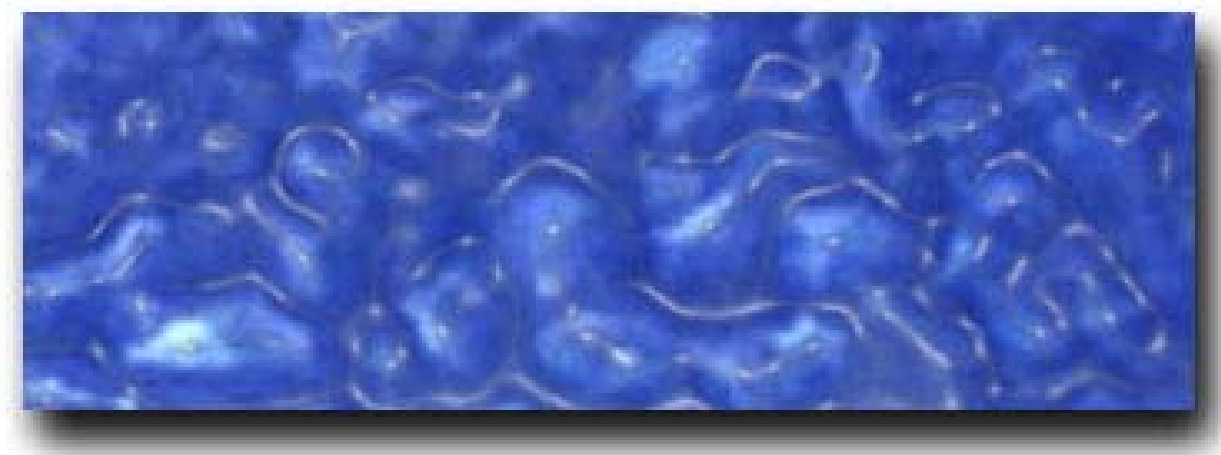

\author{
Porous Substrate
}

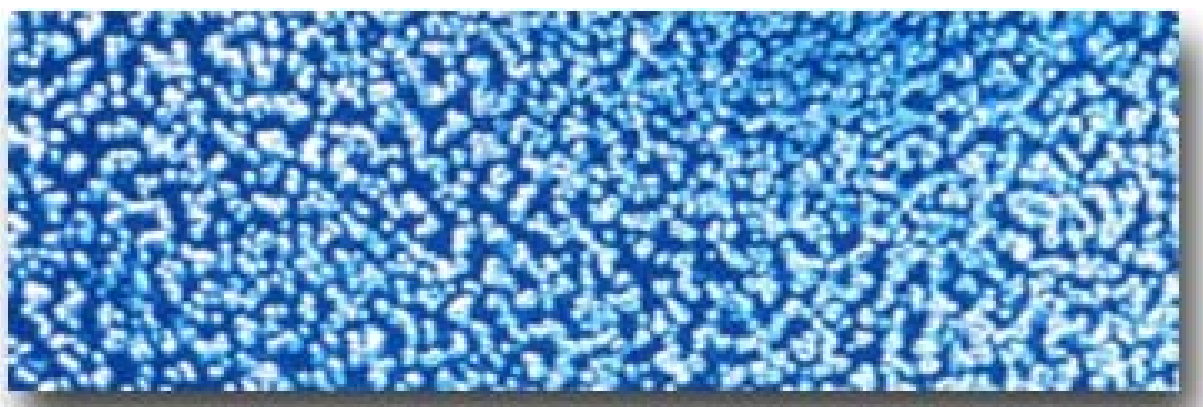

Dense Substrate

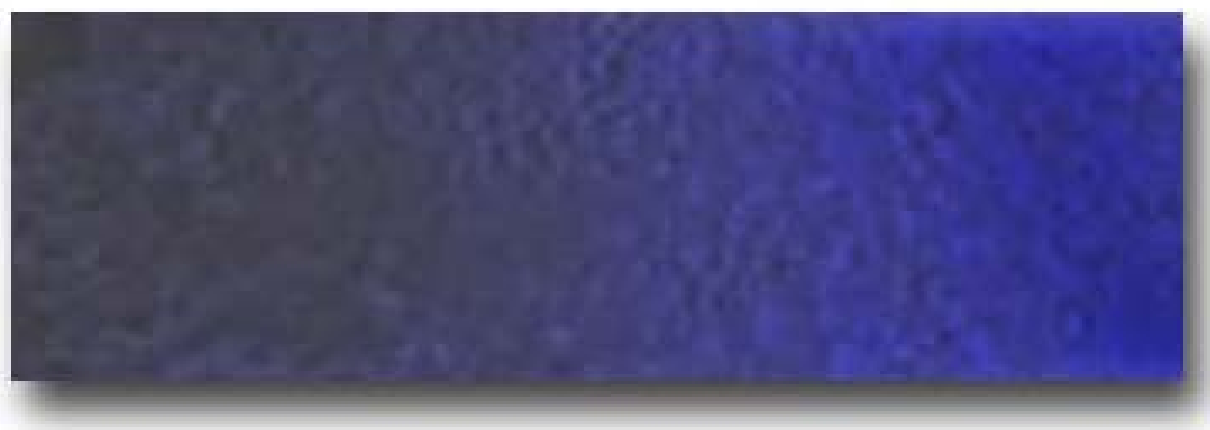

Polymerized Liquid 


\section{Constant Pressure Flux Measurements}

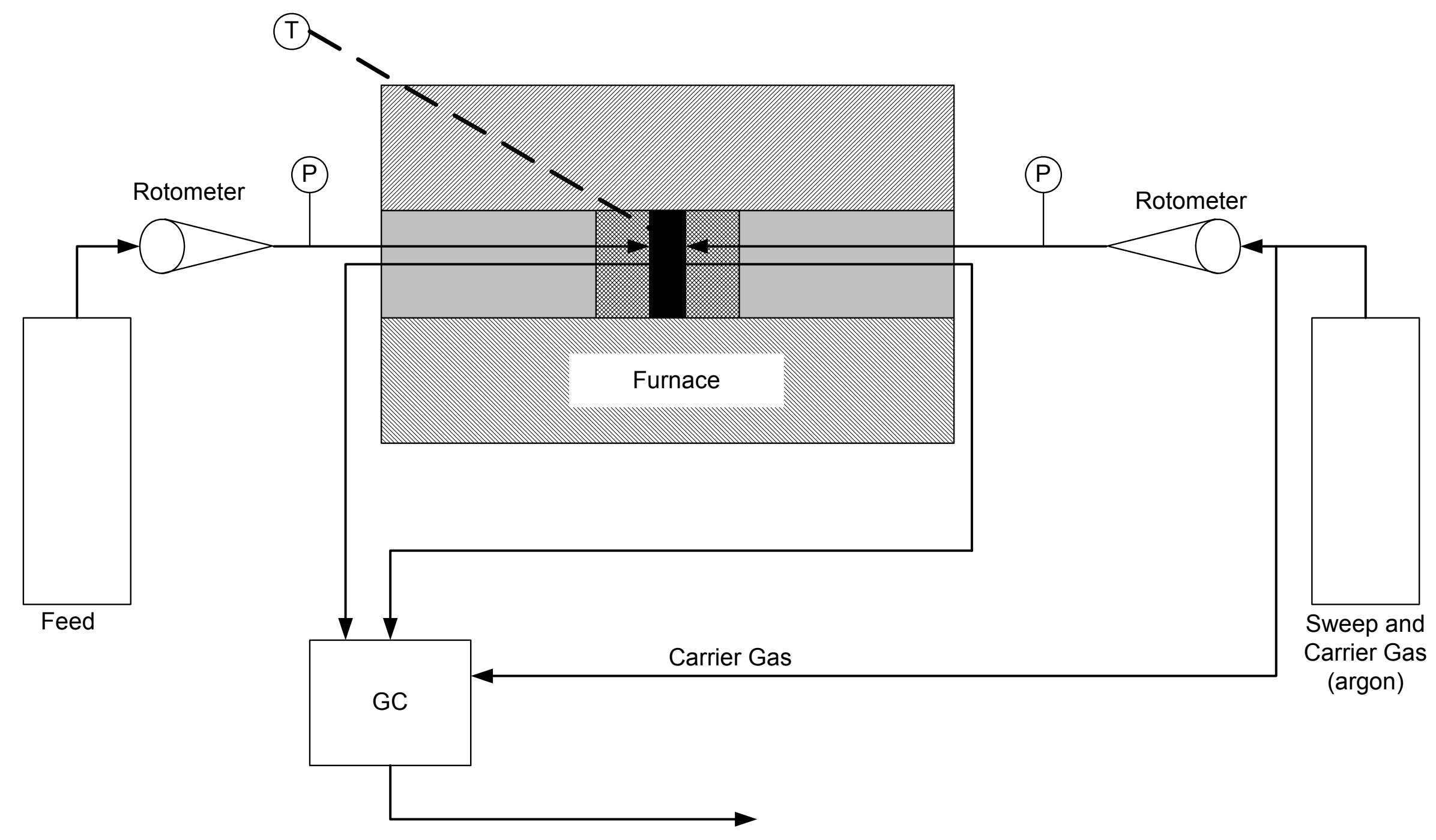




\section{Developmental Progression}

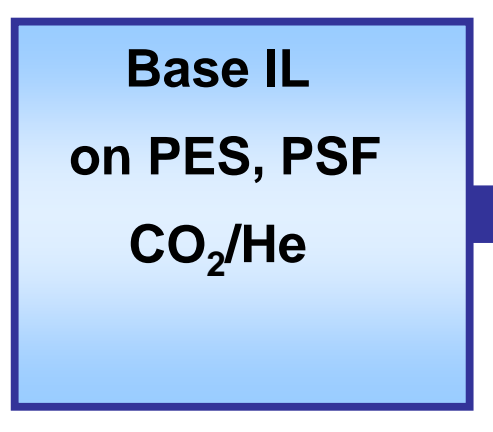

Proof of Concept

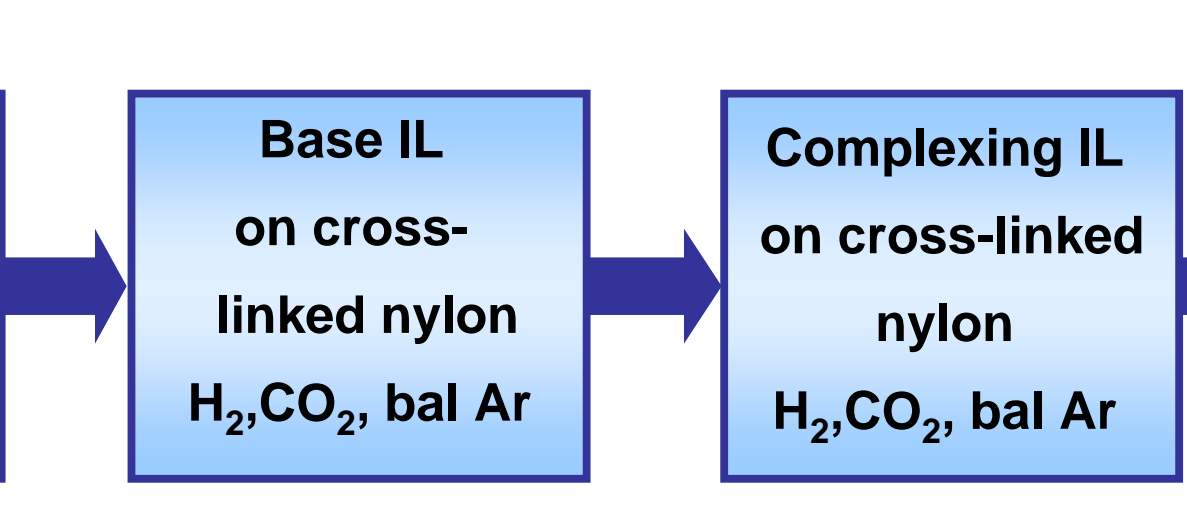

Support Development
Facilitated Transport Development
Base IL on crosslinked nylon Complexing IL on cross-linked nylon $\mathrm{H}_{2}, \mathrm{CO}_{2}, \mathrm{CO}$, bal $\mathrm{Ar}$ $\mathrm{H}_{2}, \mathrm{CO}_{2}, \mathrm{H}_{2} \mathrm{~S}$, bal

Contaminant Testing 


\section{Support Failure Limits Performance}

[HMIM] [Tf $\left.{ }_{2} \mathrm{~N}\right]$ on PSF

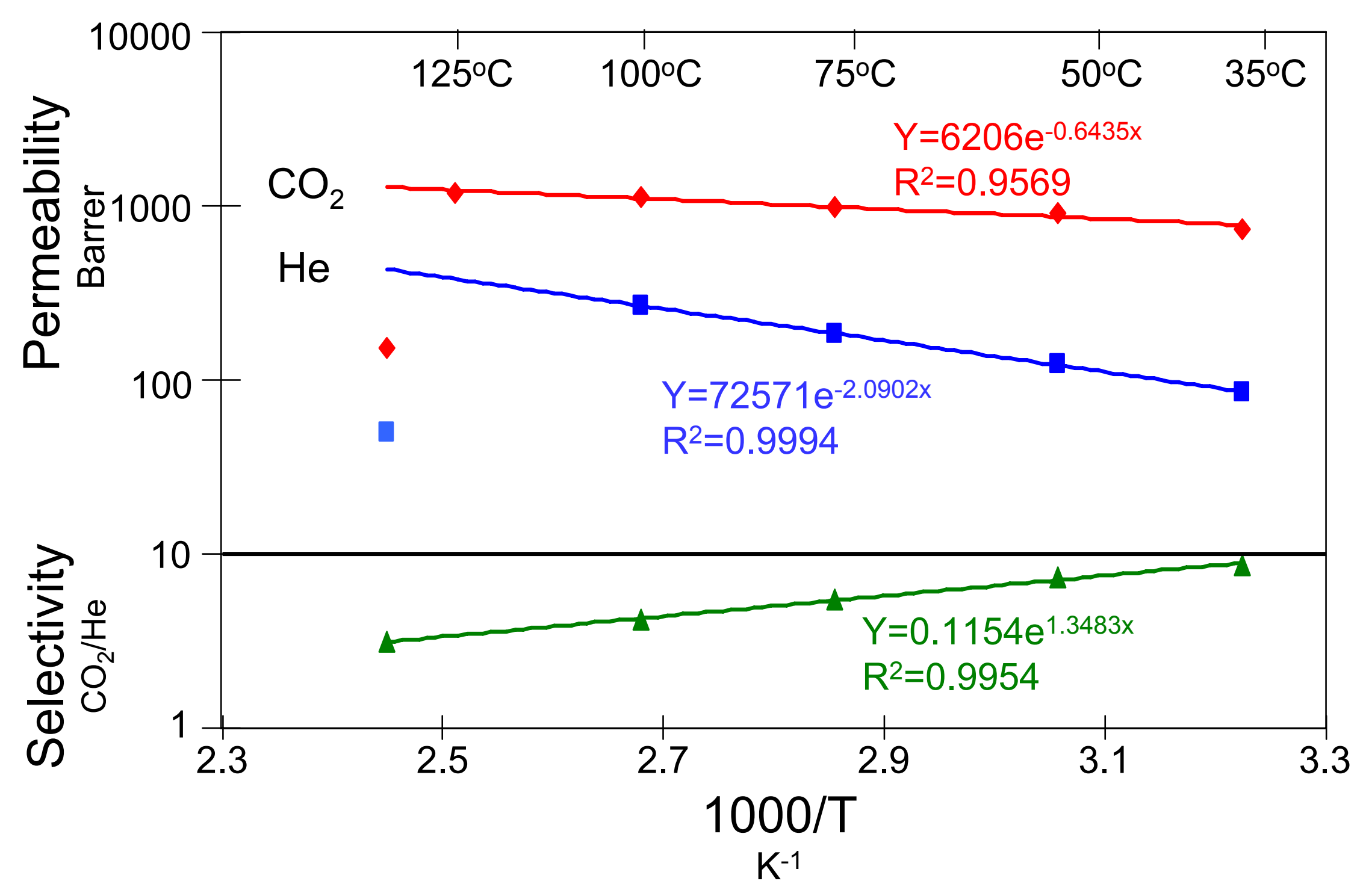




\section{Cross-linking Stabilizes Support}

$[\mathrm{HMIM}]\left[\mathrm{Tf}_{2} \mathrm{~N}\right]$ on Cross-linked Nylon

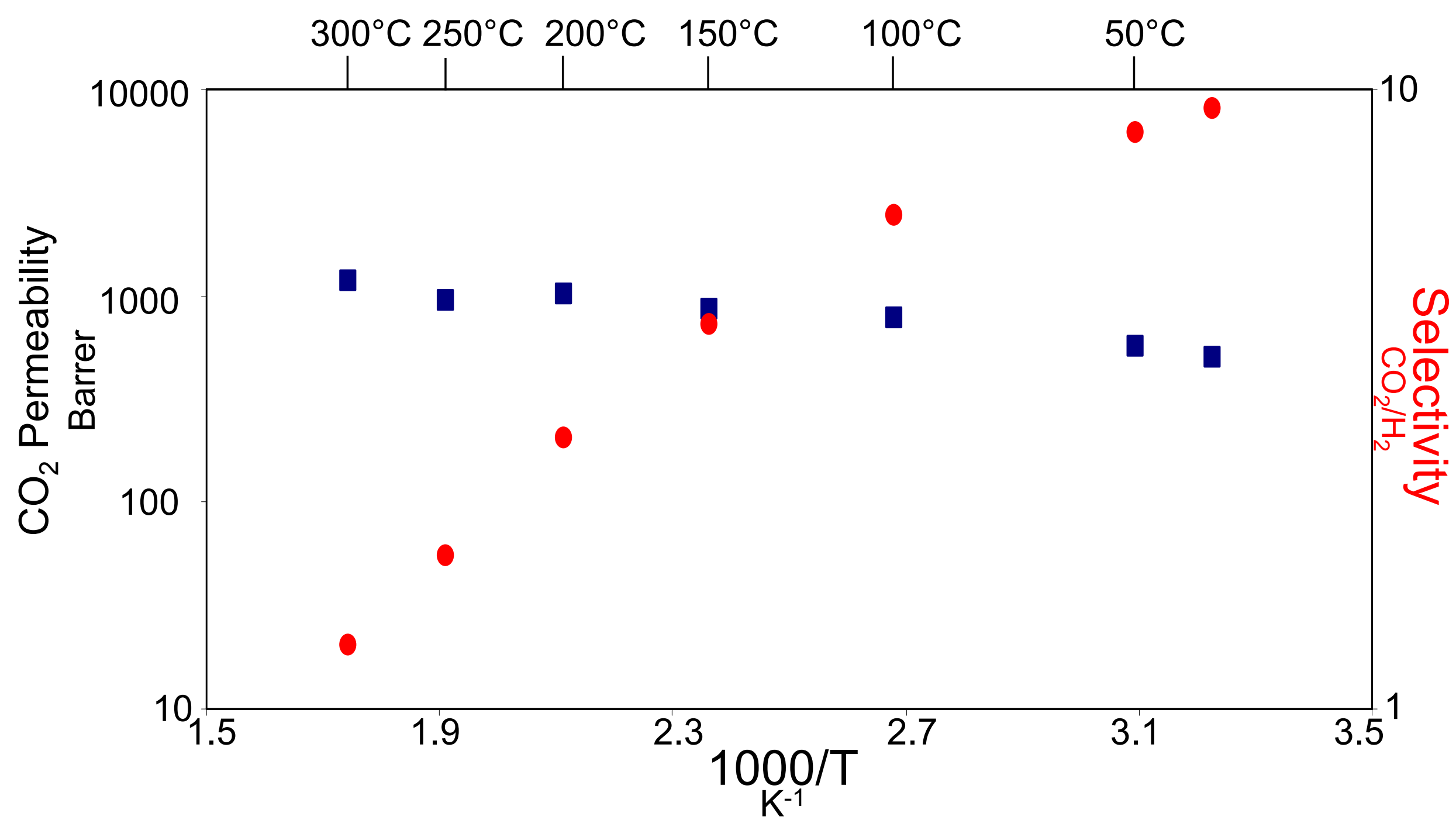




\section{Loss of Selectivity at High Temperature: A Problem of Mechanism}

Permeability $=$ Solubility $X$ Diffusivity

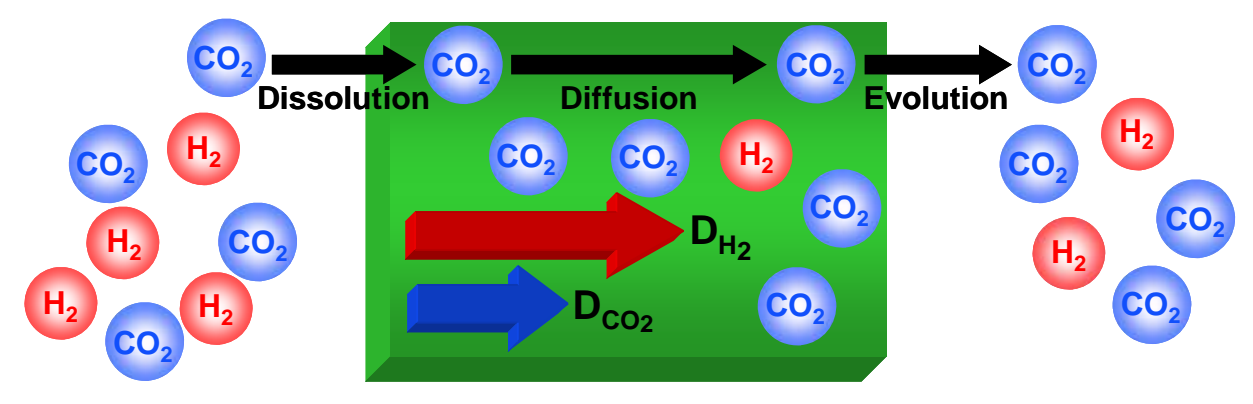

Selectivity $=\frac{\mathrm{CO}_{2} \text { Permeability }}{\mathrm{H}_{2} \text { Permeability }}$

Solution Diffusion 


\section{Formation of Chemical Complexes: A Potential Solution}
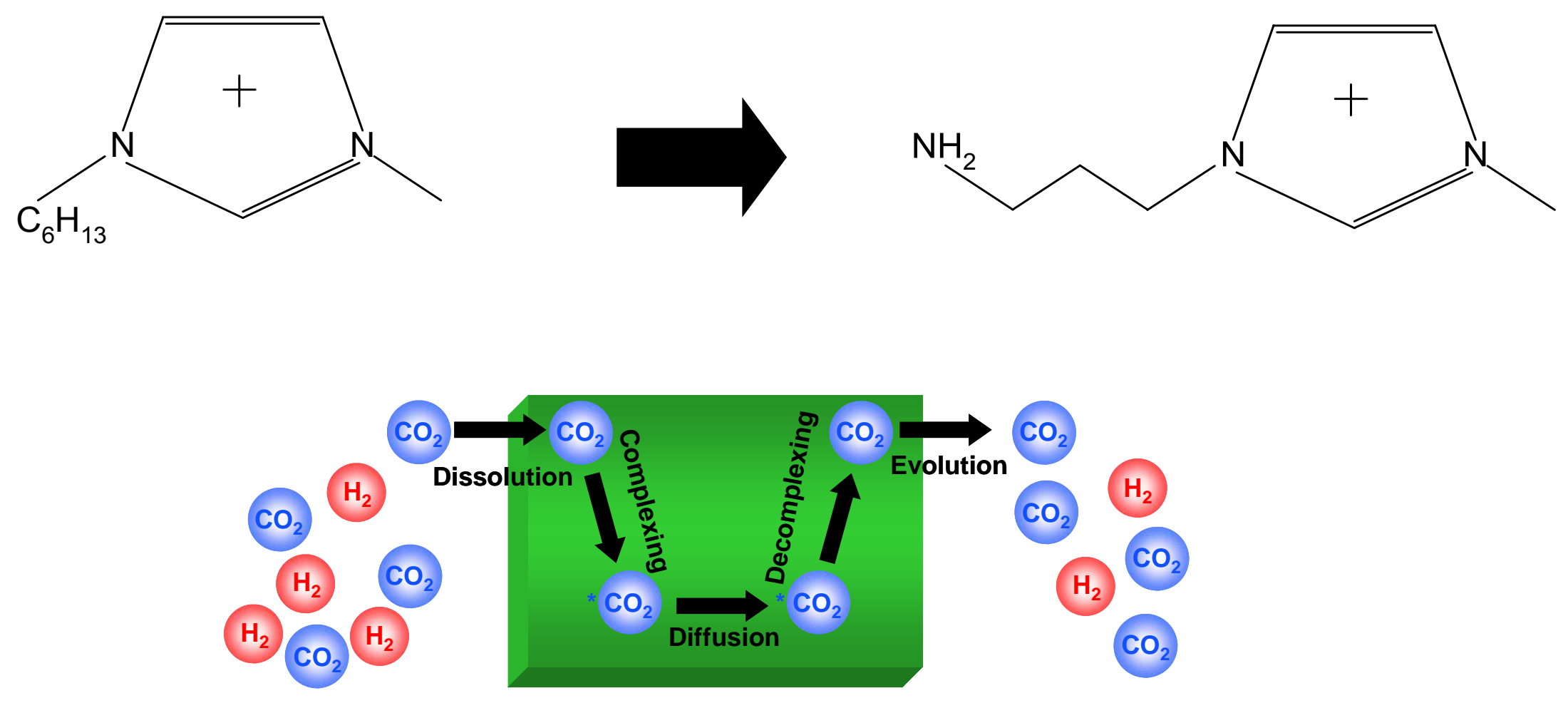


\section{Facilitated Transport Increases Performance}

$\left[\mathrm{NH}_{2} \mathrm{PMIM}\right]\left[\mathrm{Tf}_{2} \mathrm{~N}\right]$ on Cross-linked Nylon

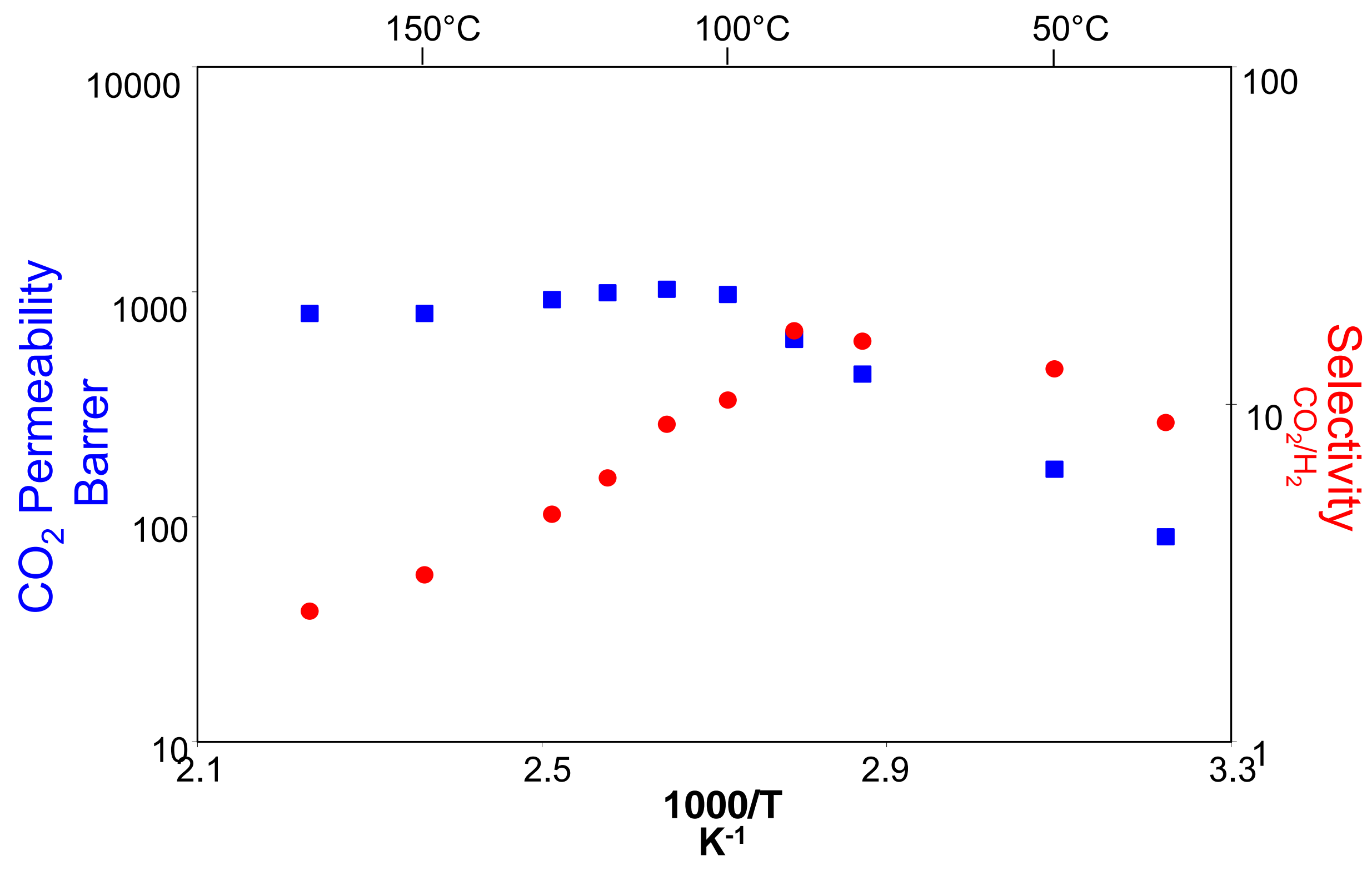




\section{Other Syngas Constituents Must be Considered}

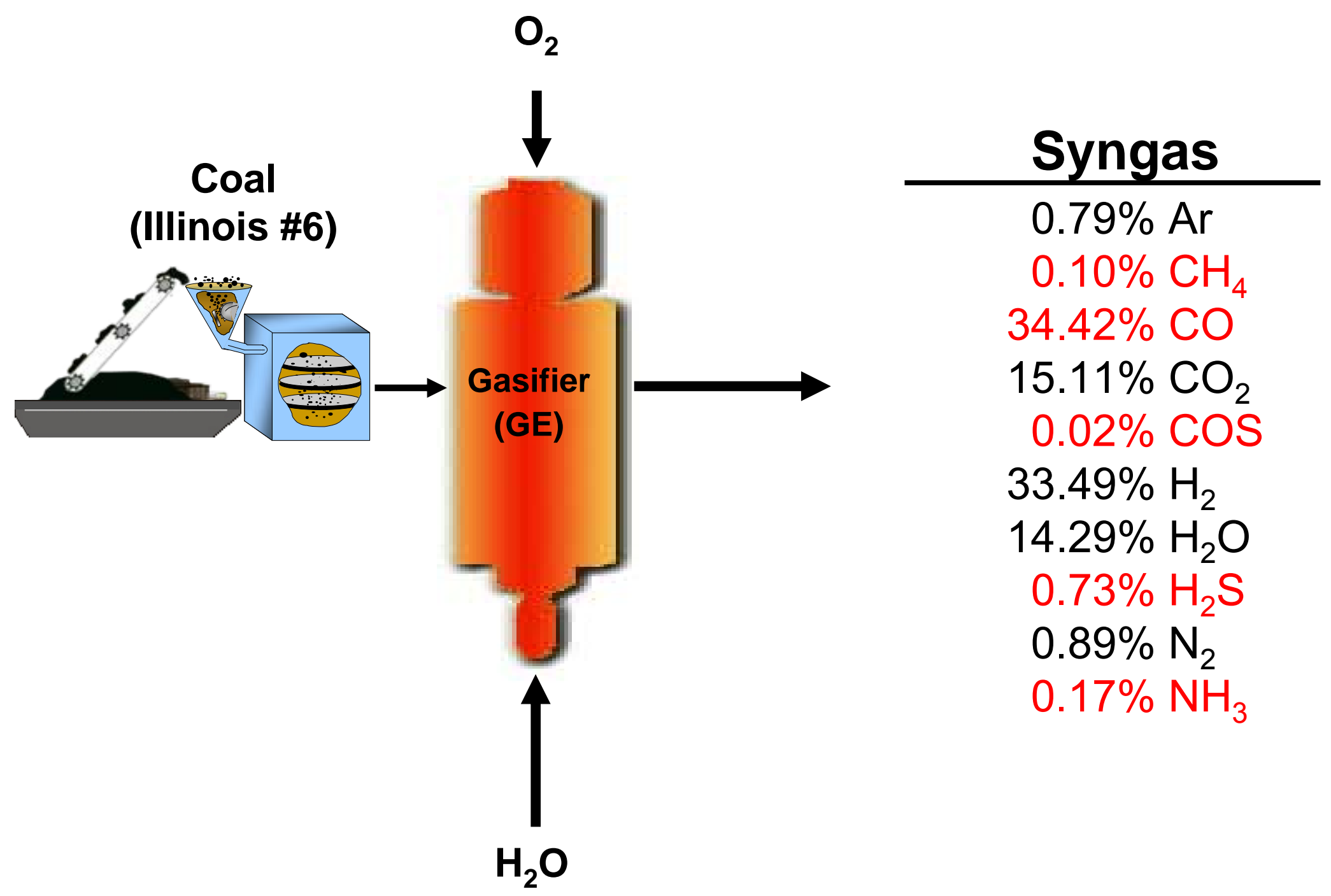




\section{CO Increases $\mathrm{CO}_{2}$ Permeability}

$[\mathrm{HMIM}]\left[\mathrm{Tf}_{2} \mathrm{~N}\right]$ on Cross-linked Nylon

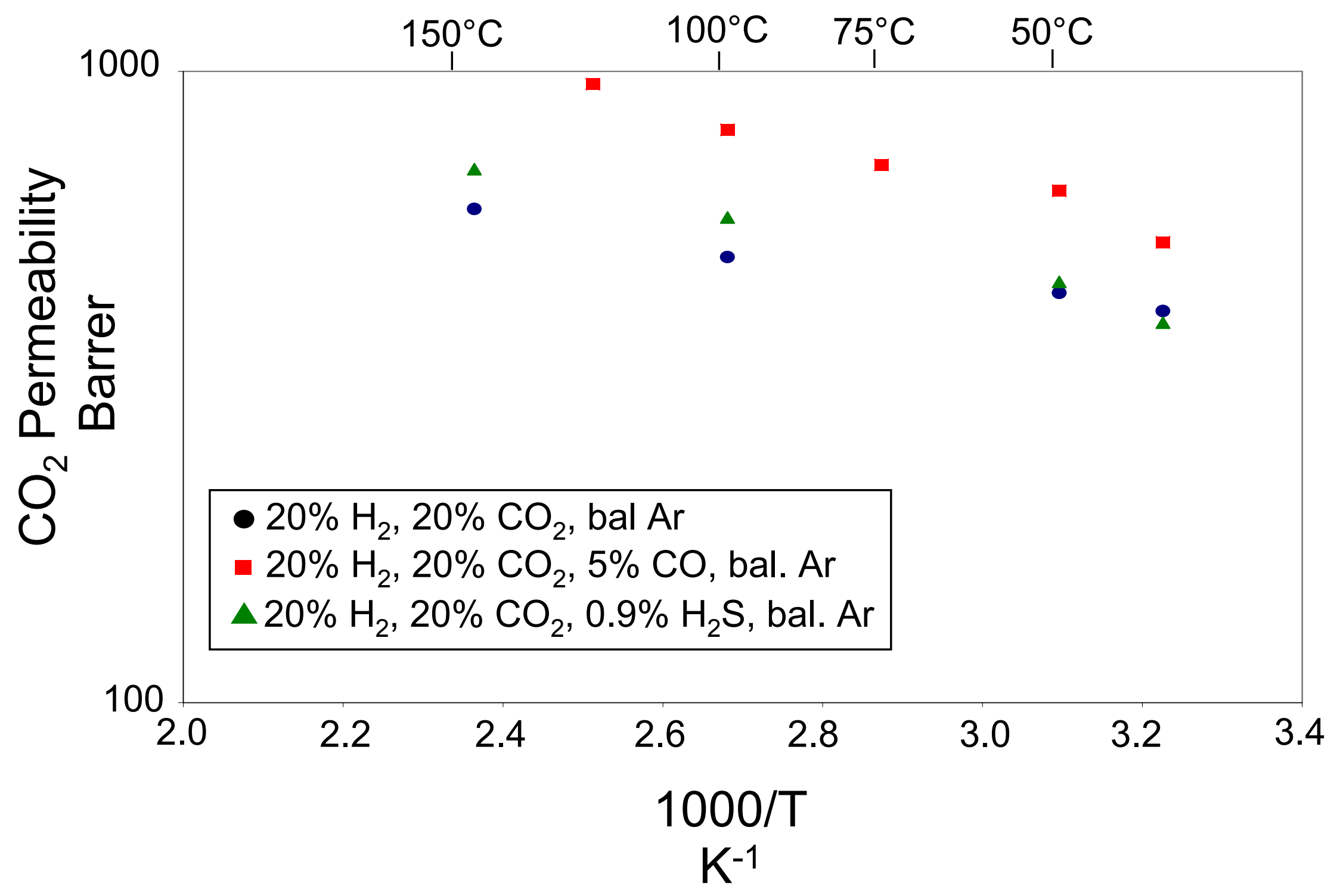




\section{Contaminants Eliminate Facilitated Transport}

$\left[\mathrm{NH}_{2} \mathrm{PMIM}\right]\left[\mathrm{Tf}_{2} \mathrm{~N}\right]$ on Cross-linked Nylon

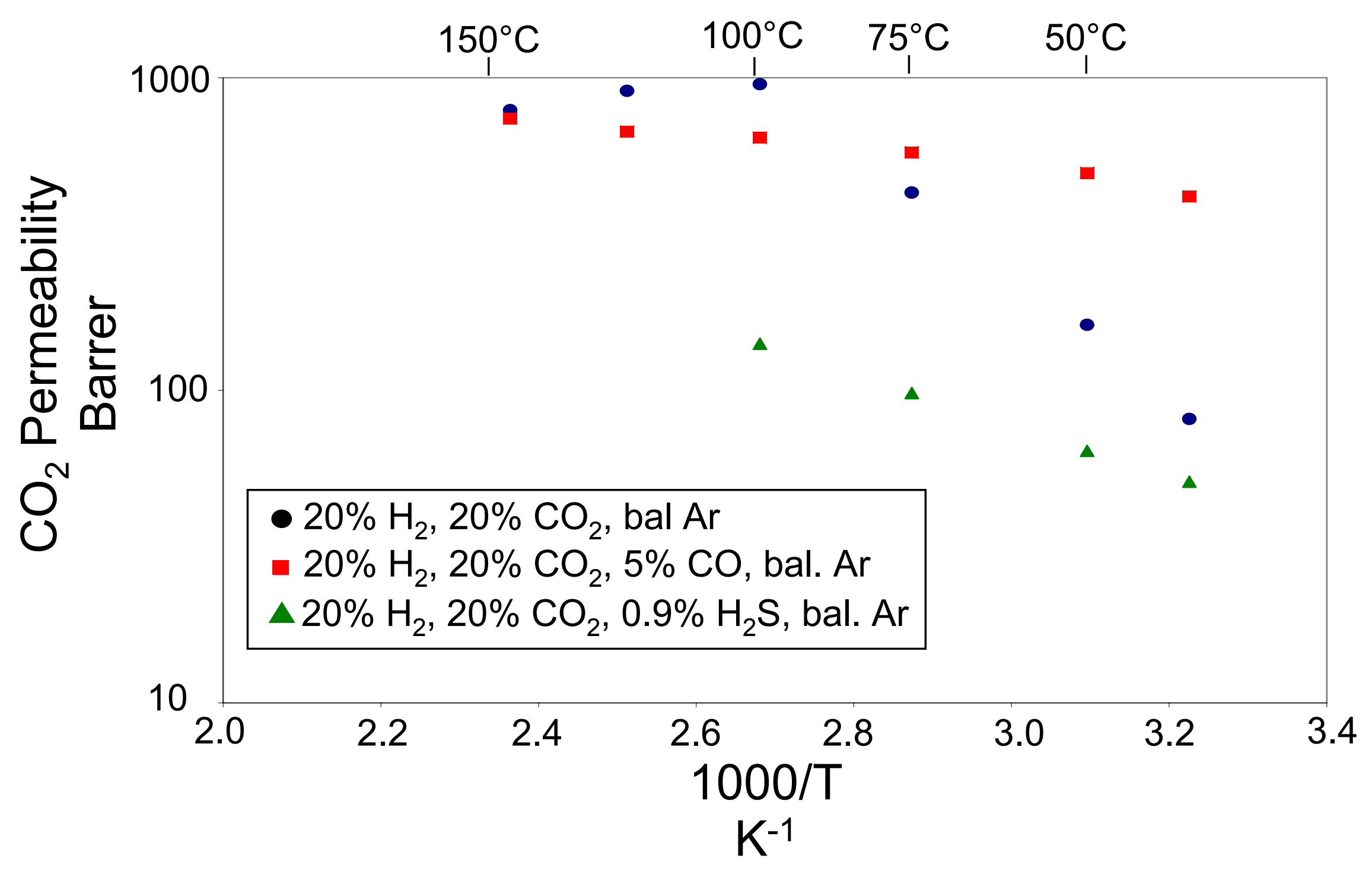




\section{Contaminants Eliminate Facilitated Transport}

$\left[\mathrm{NH}_{2} \mathrm{PMIM}\right]\left[\mathrm{Tf}_{2} \mathrm{~N}\right]$ on Cross-linked Nylon

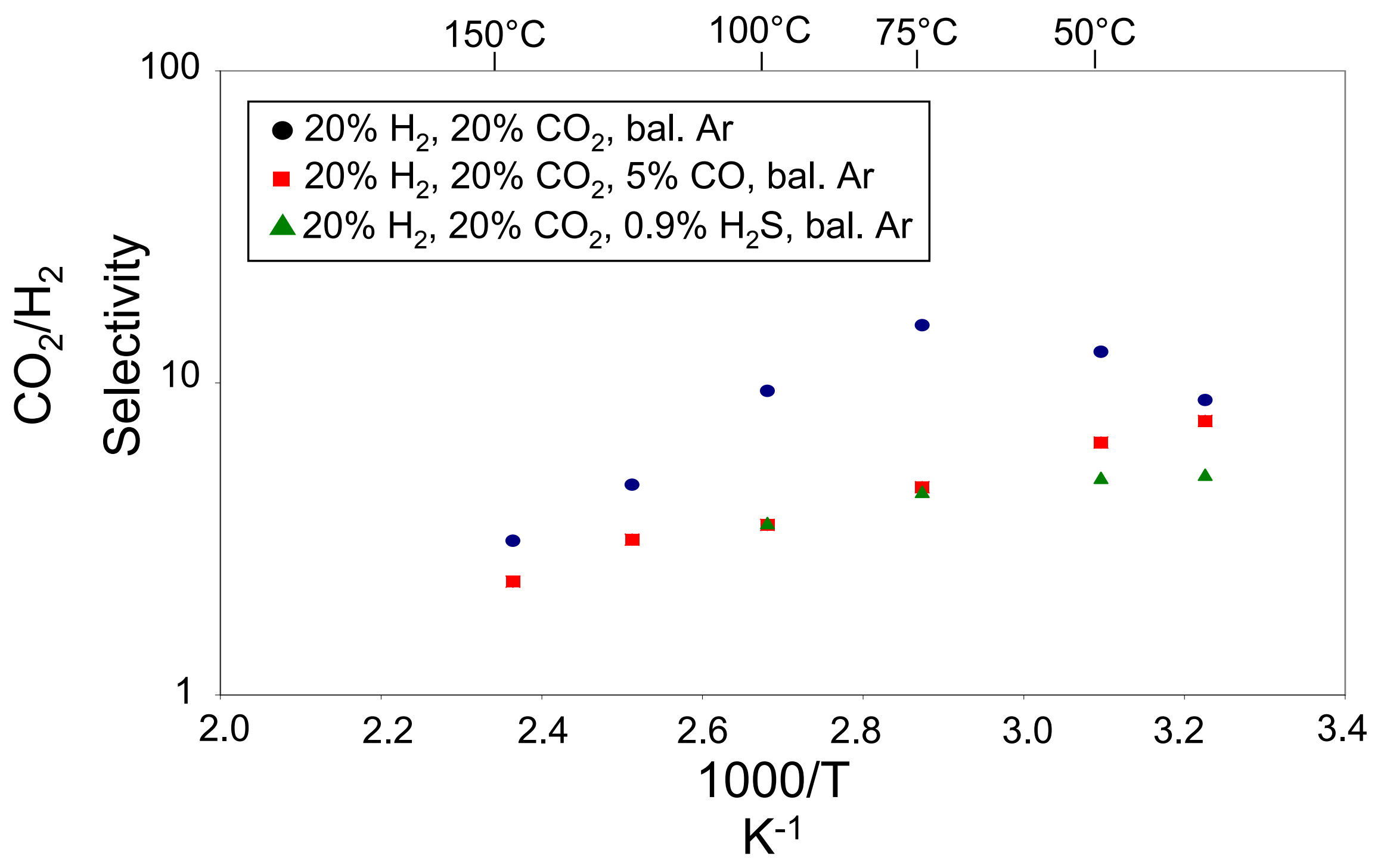




\section{Hypothesis: Contaminants Interfere with Complex Formation}

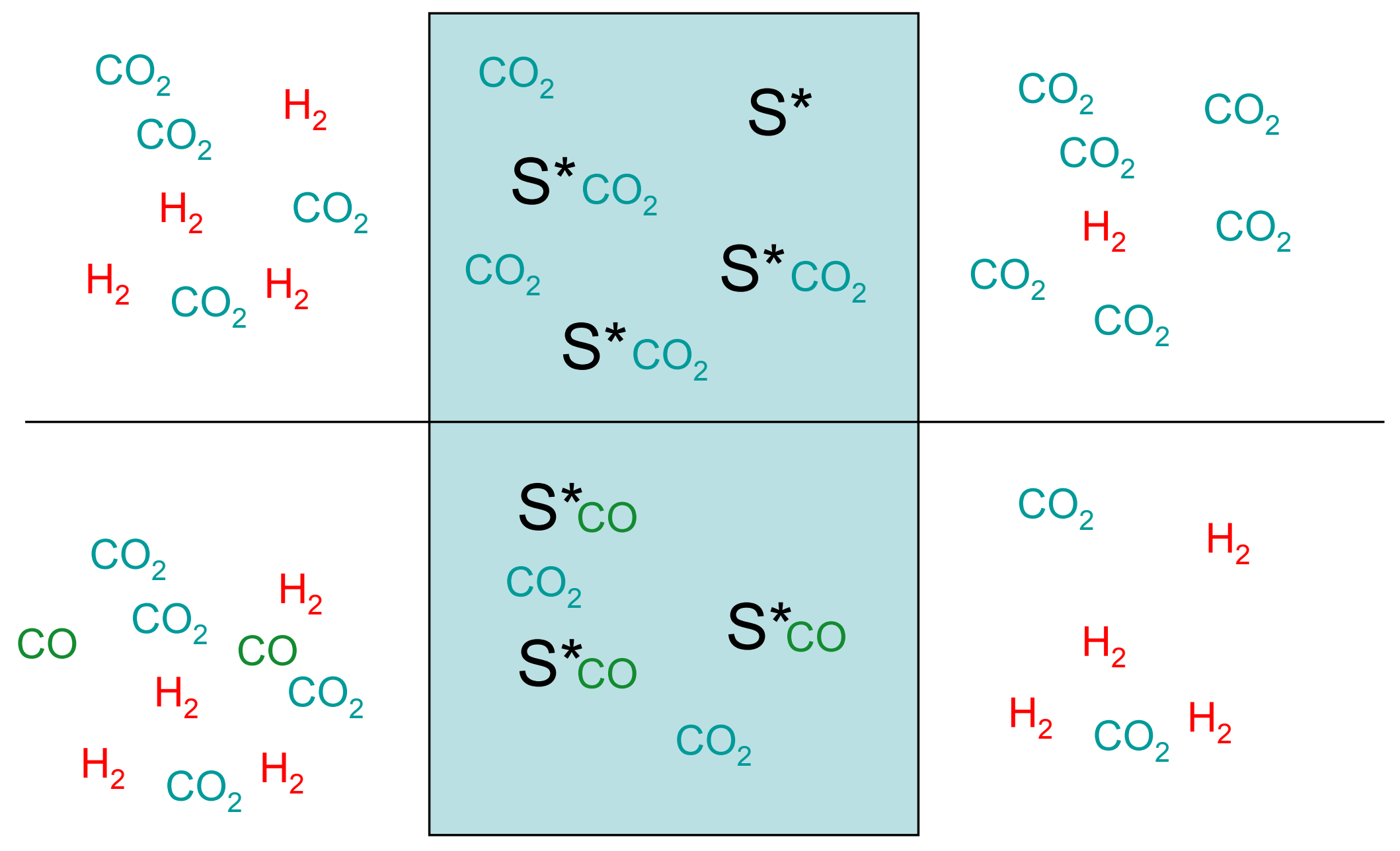




\section{Summary}

- High temperature $\mathrm{CO}_{2}$ selective membranes may facilitate water-gas shift and enhance IGCC efficiency

- Ionic liquid membranes with cross-linked supports may be employed at water-gas shift conditions

- Fabrication of temperature-stable facilitated transport membranes is possible with ionic liquid transport media

- Facilitated transport membranes based on amine complexes show significant vulnerability to $\mathrm{H}_{2} \mathrm{~S}$ and $\mathrm{CO}$

- Other complexes will be explored 


\section{Acknowledgement}

The authors gratefully recognize the Brennecke and Maginn research groups at the University of Notre Dame for their efforts in the synthesis and characterization of the ionic liquids along with their invaluable expertise in these areas. They have been instrumental in the development of new ionic liquids for this study. We look forward to further rewarding collaboration. 